\title{
Parameter Tuning of JXTA-based P2P Platforms
}

\author{
Fatos Xhafa \\ Dept. of Languages and Informatics Systems \\ Technical University of Catalonia \\ Campus Nord, C/Jordi Girona 1-3 \\ 08034 Barcelona, Spain \\ EMail:fatos@lsi.upc.edu \\ Jordi Missé Bertran \\ Dept. of Languages and Informatics Systems \\ Technical University of Catalonia \\ Campus Nord, C/Jordi Girona 1-3 \\ 08034 Barcelona, Spain \\ EMail: jmisse@lsi.upc.edu
}

\begin{abstract}
P2P technologies have appeared as disruptive technologies that have spawned large scale collaborative applications involving thousands or even millions of users world-wide. Undoubtedly, most successful applications of $P 2 P$ paradigm are information sharing between individuals and groups of users, which enable sharing and searching for digital content information, and exchanging via P2P communication such information. However, P2P applications are still difficult to program and to efficiently deploy in real network infrastructures and usually have network efficiency problems. A part from intrinsic difficulties of $P 2 P$ algorithmics, there are difficulties in efficiently tuning $P 2 P$ applications in real environments. Indeed, $P 2 P$ applications usually involve many parameters, whose values cannot be arbitrarily fixed but require a careful tuning to achieve expected performance. In this work, we show by example of a P2P JXTA-based platform, how to tune the parameters of the platform using existing software tools (JConsole and JHat) and enhance its performance.
\end{abstract}

\section{Introduction and motivation}

P2P systems [9], [8], [5] appeared as the new paradigm after client-server and web-based computing.

\author{
Zorion Arrizabalaga \\ Dept. of Languages and Informatics Systems \\ Technical University of Catalonia \\ Campus Nord, C/Jordi Girona 1-3 \\ 08034 Barcelona, Spain \\ EMail: zarrizabalaga@lsi.upc.edu \\ Leonard Barolli \\ Dept. of Information and Communication Eng. \\ Fukuoka Institute of Technology \\ 3-30-1 Wajiro-higashi, Higashi-ku \\ Fukuoka 811-0295, Japan \\ EMail: barolli@fit.ac.jp
}

P2P systems are quite popular systems to share huge amount of data such as Napster, Gnutella, FreeNet and others. One of the main characteristics of such systems is file sharing among peers. However, the improvement of $\mathrm{P} 2 \mathrm{P}$ protocols is enabling the development of $\mathrm{P} 2 \mathrm{P}$ applications others than the well-known file-sharing applications. However, there is still few work to bring P2P system to real word applications, mainly due to the lack of robust $\mathrm{P} 2 \mathrm{P}$ platforms that would allow the deployment of large P2P systems. The JXTA platform [4], [6], [7] is making possible the development of $\mathrm{P} 2 \mathrm{P}$ real-world applications.

$\mathrm{P} 2 \mathrm{P}$ applications are still difficult to program and to efficiently deploy in real network infrastructures and usually have network efficiency problems [3]. A part from intrinsic difficulties of $\mathrm{P} 2 \mathrm{P}$ algorithmics, there are difficulties in efficiently tuning $\mathrm{P} 2 \mathrm{P}$ applications in real environments. Indeed, P2P applications usually involve many parameters, whose values cannot be arbitrarily fixed but require a careful tuning to achieve expected performance. This is precisely the motivation of this work; we address the issue of how to tune the parameters of $\mathrm{P} 2 \mathrm{P}$ platforms using existing software tools, such as JConsoloe and JHat $\mathrm{t}$ enhance their performance. We exemplify the approach through a P2P JXTA-based platform, namely, JXTA-Overly platform [10], [11]. Although most current research effort are devoted to tuning network applications, testbed and 
configuration issues of deployment of $\mathrm{P} 2 \mathrm{P}$ systems are attracting the interest of researchers [1], [2].

The rest of the paper is organized as follows. We give an overview of the JXTA library in Section 2. The design of the JXTA-Overlay is given in Section 3. In Section 4 we give a description of most important parameters of JXTA-Overlay P2P platform and their tuning. Finally, we conclude in Section 5 with some remarks and indicate directions for future work.

\section{JXTA networks}

JXTA library is a set of open protocols proposed by Sun Microsystems that allow any connected device on the network ranging from cell phones and wireless PDAs to PCs and servers to communicate and collaborate in a P2P manner (see e.g. [4], [7]). JXTA peers create a virtual network in which any peer can interact with other peers and resources directly even when some of the peers and resources are behind firewalls and NATs. We describe next the main entities of JXTA networks.

Peer: Any interconnected node is called peer. Peers work independently and asynchronously with other peers. Peers publish one or more interfaces that are used by other peers to establish peer-to-peer connections.

PeerGroup: A PeerGroup is a collection of peers that are joined to provide a secure shared environment for the participating peers. PeerGroups can decide their own policy of peer membership. Note that peers can simultaneously belong to more than one PeerGroup. As in the case of peers, PeerGroups offer their services such as discovery services, pipe services, peer join services, monitoring services etc.

Pipes: A pipe is a virtual communication channel established between two processes. A computer connected to the network can open, at transport level, as many pipes as its operating system permits. These channels act as data links between the two communication points. Through pipes can be sent any types of objects such as strings, binary code, java objects, etc. JXTA offers both unidirectional, not secure pipes, and bidirectional secure pipes.

Messages: Messages are objects used for communicating and interchanging data. A message is essentially an ordered sequence of tags/values, which could also include binary code (appropriately encoded).

Advertisements: The JXTA resources/services are represented using advertisements. An advertisement is a meta-data structured information (XML document), which are published with a certain lifetime that specifies its availability. JXTA offers the following types of advertisements: Peer Advertisement, Peer Group Advertisement, Pipe Advertisement, Module Class Advertisement, Module Spec Advertisement, Module Impl Advertisement, Rendezvous Advertisement and Peer Info Advertisement.

Further, peers can be classified in Limited Edge peer (peers that can send and receive messages but cannot send advertisements neither store advertisements in its cache); Complete Edge peer (peers that can send/receive messages, store advertisements but cannot manage resource discovery advertisements); Rendezvous peer (besides functionalities mentioned above, Rendezvous peers can manage resource discovery advertisements. Each group must have at least a rendezvous; when a peer joins a group of peers, it automatically looks for a rendezvous in the group. Moreover, rendezvous keep a list of other rendezvous and their respective peers); and, Relay peer (this type of peer serves as an intermediate nodes. Relay peer keep routing information to other peers and sends messages to other peers that can't access directly to another peer.)

\section{The JXTA-Overlay}

The objective of the overlay is to provide a set of basic primitives for file sharing, the discovery and allocations of resources, instant messaging, etc. This set of primitives is intended to be as complete as possible regarding the functionalities needed in most P2P applications. An important characteristics of the primitives is their independence from the applications that will be using them. Moreover, the primitives allow to keep the intrinsic decentralized nature of P2P systems.

\subsection{The primitives}

The set of primitives includes functionalities that allow peer discovery, peer's resources discovery, resource allocation, file/data sharing, discovery and transmission, instant communication and peer group functionalities, among others.

The primitives are organized in interfaces according to an affinity criterion. In what follows we give the main functionalities of these interfaces; here we use indistinctly the terms peer and resource.

Authentication: This interface includes typical methods for authentication of the final user/application that will be using the resources managed by the overlay. It should be noted that another authentication could be established at the application level, which would be independent of the overlay. In 
this interface we have, among others, the methods connect (which verifies the authentication by calling the verifyAuthentification method of JXTA, connects to a broker, flushes the local cache and fires an event); configure (which configures the local cache; it is called just once at the beginning); login and disconnect.

Resource discovery and information: This interface includes functionalities related to the discovery of peers managed by the overlay. The implementation of these functionalities is later done by using JXTA discovery services. It should be noted that, as part of resource discovery, the overlay includes functionalities to discover a resource of certain desired characteristics. Thus we have, among others, the methods discoveryEvent, getPeerName and getPeerID.

Management of executable tasks: An important place in the primitives is given to functionalities related to the management of executable tasks in distributed applications. These functionalities are intended to give service to users/applications on top of the overlay that submit executable tasks and receive results in turn. Thus we have, among others, the following methods:

- executableTaskRequestRandom: a new executable task is requested to be executed in another peer selected at random.

- executableTaskRequestselectedpeer: a new executable task is requested to be executed in a (group of) selected peer(s).

- executableTaskRequestDataEvaluator: evaluates the execution of a task according to task data/characteristics.

- executableTaskRequestEconomicEvaluator: evaluates the execution of a task according to a given economic model based on task data/characteristics.

- executableTaskDel: deletes a task from list of pending tasks.

- executableTaskAccepted: indicates acceptance of a task execution in a specified resource.

- executableTaskDeny: indicates deny of a request for a task execution

- executableTaskFinished: advertises that the execution of task has successfully been finished.

- executableTaskCanceledRequest: requests cancellation of task execution.

- executableTaskCanceledByDestination: indicates that the executable task has been cancelled at destination resource.

- executableTaskCanceledBysender: indicates that the executable task has been cancelled by task's sender.

- addExecutableType: adds a new type of executable tasks that a resource supports. Similarly there is the delExecutableType method.

- addExecutableTypes: adds a list of executable types that a specified group of resources can support. Similarly there is the delExecutableTypes method.

- getExecutableTaskLocal Info: returns the local information about an executable task.

- getExecutableTaskRemoteInfo: returns the information about an executable task at a remote peer (including the prediction for the completion time of the task).

- getTasks: returns all pending tasks of a specified type (e.g. executable task, file transfer, etc.).

File sharing, discovery and transmission: This includes sharing, discovery and transmission of files, which are basic functionalities of the overlay. The objective of these functionalities goes beyond the sharing in $\mathrm{P} 2 \mathrm{P}$ systems since file transmission is necessary for submitting tasks to resources. Thus we have, among others, the following methods: addSharedFile, addSharedDirectory, delsharedFile, getsharedFiles, sendFileAccepted, sendFileDeny, sendFileGroup, findFile, fileRequestRandom, fileRequestselectedPeer,

fileRequestEconomicEvaluator, cancelTransfer, local InfoTransfer.

Instant communication: This interface includes methods for instant communication between peers and include sendMsgPeer (for sending a message to a specified peer) and sendMsgGroup (for sending a message to a group of peers).

Peer's statistics: Statistic information of resources is relevant for applications that will be built on top of the overlay. Thus we have, among others, the following methods:

- getPeerstatistics: returns statistic information on a specified resource.

- getGroupstatistics: returns statistic information on a group of resources.

- getBrokerstatistics: returns statistic information on a specified broker resource.

- getBrokerstatistics: returns statistic information on the broker of a specified group of resources.

- getClientstatistics: returns statistic information on a specified edge peer.

- getClientstatistics: returns statistic information on a specified group of edge peers.

There are also some primitives related to the peer's local cache that we have omitted here.

\subsection{Peers in JXTA-Overlay}

The set of the primitives is implemented by taking advantage that JXTA allows different types of peers. In the overlay we have defined client peers and broker peers. The former are complete edge peer while the later act as rendezvous and relays. Any PeerGroup has at least a broker to which client peers get connected 
and send their requests (e.g. resource allocation petitions).

Broker peers: The mission of a broker node is to manage and control all requests received from client peers. Also, broker manages the events produced by such requests and propagates them to superior levels of overlay. Among broker's functionalities we distinguish: event management, controlling the resources connected to the broker, maintaining the organization of resources in groups, finding the best resource for file sharing, finding the best resource for executing submitted tasks, maintaining updated statistic information, etc.

Client peers: Client peers can be of two types: CMDClient, which is instantiated by peer that do not interact with final user and GUIClient that offers a graphical interface for the final user.

\section{Parameter Tuning}

In this section we first present the most relevant parameters in JXTA-based P2P platforms and then show how to use existing software, such as JConsole and Jhat, for their tuning.

\subsection{JXTA-Overlay parameters}

The parameters in JXTA-Overlay can be divided into two groups: parameters related to Broker peers and parameters related to Client peers. The parameters are given in Table 1.

As can be seen from Table 1, Broker peer parameters include discovery time, publishing time (for resource discovery and rooms), advertisement time for broker's statistics and expiration time for broker's statistics. It should be noted that high values of these parameters could lead to an inconsistent state of the network; for instance, a high value of advertisement time for broker's statistics related to peer statistics could yield to obsolete information in case a peer is disconnected from the network. On the other hand, small values of the parameters could cause congestion of Broker peers due to very frequent updates.

Similarly, in case of Client peer the parameters include time parameters related to peer's advertisements, publishing, statistics, expiration as well as parameters related to pipes. Again, small values of these parameters could cause saturation of Client peers due to very frequent generation of advertisements and high traffic in the network due to the publishing and propagation of the peer's information.

These time parameters could be actually called polling-like parameters since they directly relate to the time intervals for checking the state of the network, performing actions or sending state information to the network.

Therefore, finding parameter values adjusted to the $\mathrm{P} 2 \mathrm{P}$ networks is crucial to achieve expected performance. In particular, the values of time parameters should allow the $\mathrm{P} 2 \mathrm{P}$ platform to successfully process all events and threads (executors) generated in the platform. Inappropriate values of the parameters could cause the accumulation of many events and executors that the platform might not be able to process and hence causing collapse of the platform.

\subsection{Tuning parameters of JXTA-Overlay}

JConsole $^{1}$ and JHat (Java Heap Analysis Tool) ${ }^{2}$ are standard Java tools for analyzing performance of Javabased applications. JConsole offers many functionalities for detecting the lack of memory, deadlocks as well as monitoring various threads of an application. JHat can be used in conjunction with JConsole, through its option to create a dump file (created using MBeans) that JHat can analyze the performance of the application's heap.

The performance of the JXTA-Overlay is done by analyzing Broker peers and Client peers performance (in terms of their parameters). The experimental study started with a default setting of parameters (see Tables 2 and 3) and was done in several iterating steps with the aim of finding most appropriate values of the parameters. In order to realistically measure the performance of the platform, peers shared a considerable number of files, initiated several task executions, some peers initiated file search etc. Thus, the analysis is conducted under a realistic workload of the P2P platform.

Broker peer's performance analysis. We analyzed Broker's parameters when client peers were trying to connect to the network. Using the default values of Broker's parameters, we observed that the CPU usage, heap and number of threads reach levels that can not be functional (see snapshot in Fig. 1).

The rather large number of executors could be explained because executors are created faster than Broker peer can process them (flush_cache was already applied to clear expired executors). Therefore we conducted several iterations and changed the values of parameters slowly. The final values of parameters (see Tables 2 and 3) resulted in stable values in number of threads as well in CPU and Heap usage (see snapshot in Fig. 2).

1. http://java.sun.com/javase/6/docs/technotes/tools/share/jconsole.html

2. http://java.sun.com/javase/6/docs/technotes/tools/share/jhat.html 
Table 1. Broker and Client peer parameters.

\begin{tabular}{|l|l|l|l|}
\hline Broker peer's parameters & \multicolumn{3}{|c|}{ Client peer's parameters } \\
\hline broker.discovery.time & client.ping.time & client.criteriums.adv.lifetime \\
\hline broker.discovery.publish.time & client.discovery.time & client.criteriums.adv.expiration.time & discovery.room.time \\
\hline broker.stats.adv.time & client.discovery.publish.time & client.stats.adv.time & pipe.adv.room.time \\
\hline broker.stats.adv.lifetime & client.info.adv.time & client.stats.adv.lifetime & pipe.adv.broker.time \\
\hline broker.stats.adv.expiration.time & client.info.adv.lifetime & client.stats.adv.expiration.time & pipe.adv.client.time \\
\hline broker.discovery.room.publish.time & $\begin{array}{l}\text { client.info.adv.expiration.time } \\
\text { client.criteriums.adv.time }\end{array}$ & $\begin{array}{l}\text { client.stats.update.time } \\
\text { client.stats.max.times }\end{array}$ & $\begin{array}{l}\text { pipe.adv.lifetime } \\
\text { pipe.adv.expiration.time }\end{array}$ \\
\hline
\end{tabular}

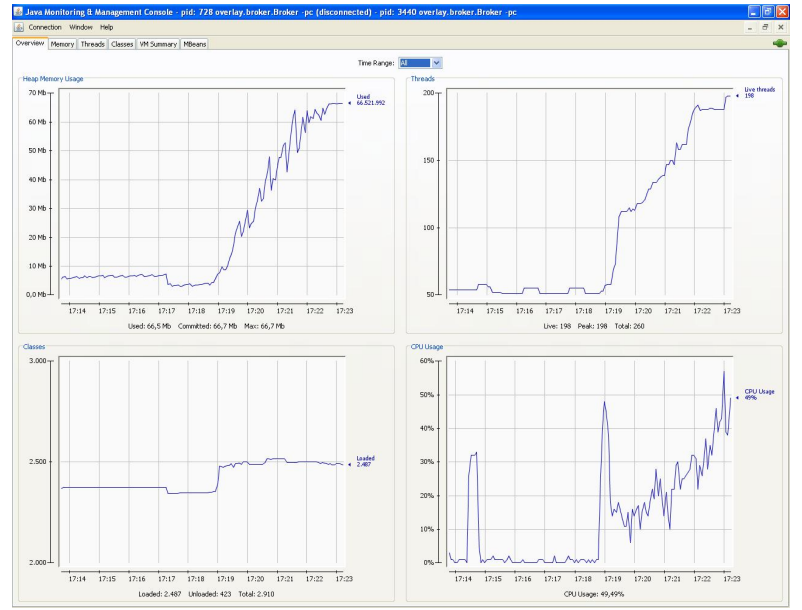

Figure 1. CPU usage, Heap and number of threads of Broker peer (initial configuration).

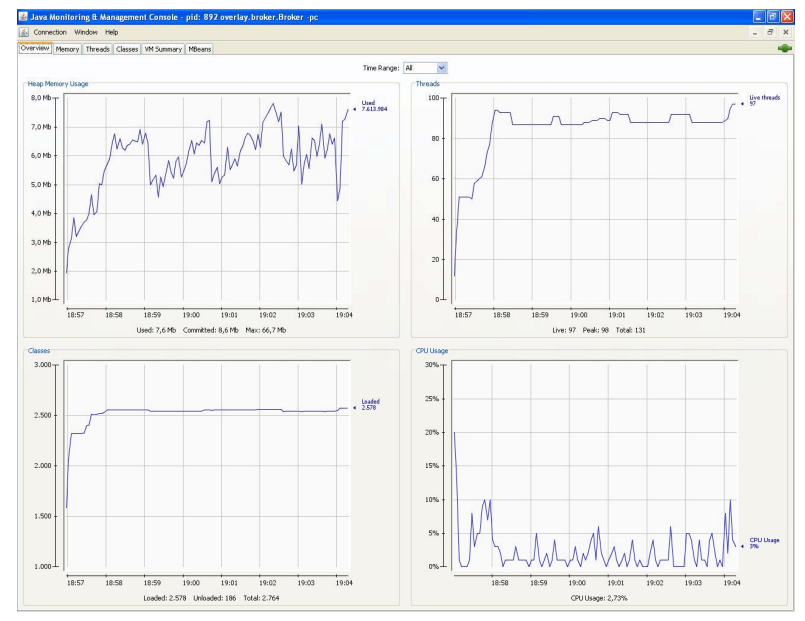

Figure 2. CPU usage, Heap and number of threads of Broker peer (final configuration).

Client peer's performance analysis. We analyzed in a similar way the performance of client peer perfor- mance. Initially we used the default configuration for client peers and monitored the CPU and Heap usage as well as number of threads. As can be seen from Fig. 3, the Heap usage and number of threads are high.

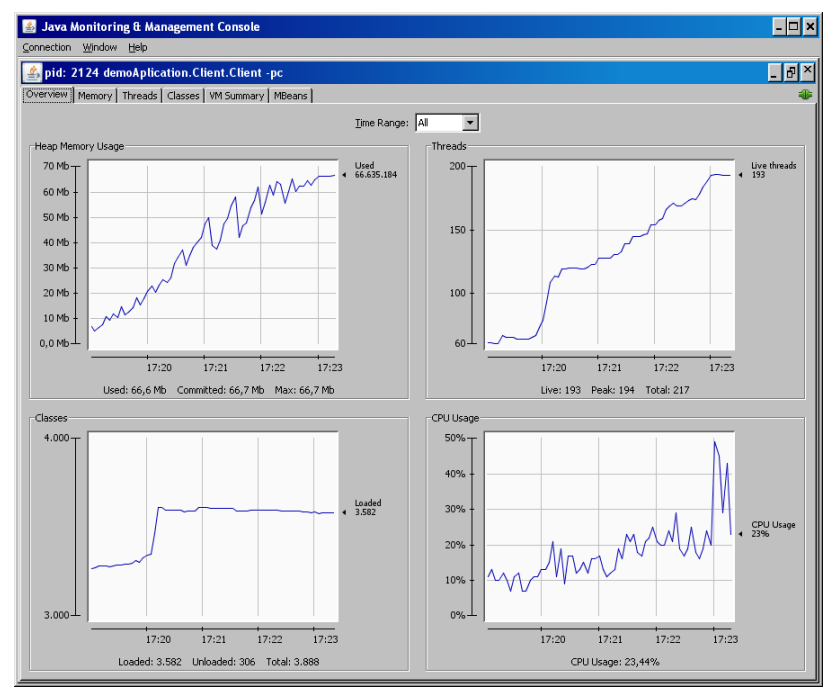

Figure 3. CPU usage, Heap and number of threads for Client peer (initial configuration).

Again, we conducted several iterations and changed the values of parameters slowly. The final values of parameters (see Table 2 and Table 3) resulted in stable values in number of threads as well in Heap usage (see snapshot in Fig. 4).

\section{Conclusions}

In this work we have addressed the issue of tuning $\mathrm{P} 2 \mathrm{P}$ applications in order to enhance their performance. $\mathrm{P} 2 \mathrm{P}$ networks involve a large number of parameters and their performance largely depends on appropriate values of the parameters. We have exemplified the approach of parameter tuning of $\mathrm{P} 2 \mathrm{P}$ platforms 


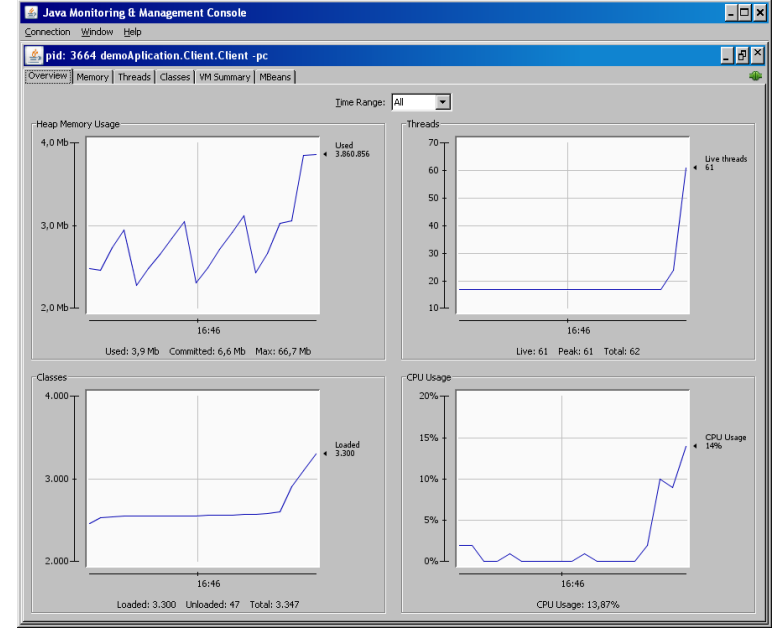

Figure 4. CPU usage, Heap and number of threads of Client peer (final configuration).

Table 2. Parameter values for Broker peer: default and new configuration.

\begin{tabular}{|l|l|}
\hline New Broker configuration & Default Broker configuration \\
\hline \# UNITS & \# UNITS \\
\hline \# In minutes: & \# In minutes: \\
\hline \# - client.ping.time & \# - client.ping.time \\
\hline \# - client.stats.update.time & \# - client.stats.update.time \\
\hline \# - client.stats.adv.lifetime & \# - client.stats.adv.lifetime \\
\hline \# - client.stats.adv.expiration.time & \# - client.stats.adv.expiration.time \\
\hline \# - executable.small.time & \# - executable.small.time \\
\hline \# - broker.stats.adv.lifetime & \# - broker.stats.adv.lifetime \\
\hline \# - broker.stats.adv.expiration.time & \# - broker.stats.adv.expiration.time \\
\hline \# In seconds: the rest & \# In seconds: the rest \\
\hline broker.discovery.time $=18$ & broker.discovery.time $=5$ \\
\hline broker.discovery.publish.time $=10$ & broker.discovery.publish.time $=5$ \\
\hline broker.stats.adv.time $=20$ & broker.stats.adv.time $=30$ \\
\hline broker.stats.adv.lifetime $=5$ & broker.stats.adv.lifetime $=5$ \\
\hline broker.stats.adv.expiration.time $=5$ & broker.stats.adv.expiration.time $=5$ \\
\hline discovery.room.publish.time $=50$ & discovery.room.publish.time $=5$ \\
\hline discovery.room.time $=50$ & discovery.room.time $=5$ \\
\hline pipe.adv.room.time $=50$ & pipe.adv.room.time $=5$ \\
\hline pipe.adv.broker.time $=10$ & pipe.adv.broker.time $=5$ \\
\hline pipe.adv.client.time $=10$ & pipe.adv.client.time $=5$ \\
\hline pipe.adv.lifetime $=25$ & pipe.advvlifetetime $=10$ \\
\hline pipe.adv.expiration.time $=50$ & pipe.adv.expiration.time $=50$ \\
\hline
\end{tabular}

through the tuning of parameters of JXTA-Overlay using existing software tools such as JConsole and Jhat. The proposed approach is useful for tuning also other important parameters such as size of PeerGroup, number of tasks and shared files in client peers in order to evaluate the scalability of the $\mathrm{P} 2 \mathrm{P}$ platform.

\section{References}

[1] D. Andersen, H. Balakrishnan, M.F. Kaashoek, and R. Morris. Experience with an evolving overlay network testbed. ACM SIGCOMM Computer Comm. Review, 33(3):13-19, 2003.

[2] B. Butnaru, F. Dragan, G. Gardarin, I. Manolescu, B. Nguyen, R. Pop, N. Preda and L. Yeh. P2PTester: a tool for measuring P2P platform performance. Proc. of Int'l Conf. on Data Engineering, 1501-1502, IEEE 2007.
Table 3. Parameter values for Client peer: default and new configuration.

\begin{tabular}{|c|c|}
\hline New Client peer configuration & Default Client peer configuration \\
\hline \# In minutes: & \# In minutes: \\
\hline \# - client.ping.time & \# - client.ping.time \\
\hline \# - client.stats.update.time & \# - client.stats.update.time \\
\hline \# - client.stats.adv.lifetime & \# - client.stats.adv.lifetime \\
\hline \# - client.stats.adv.expiration.time & \# - client.stats.adv.expiration.time \\
\hline \# - executable.small.time & \# - executable.small.time \\
\hline \# - broker.stats.adv.lifetime & \# - broker.stats.adv.lifetime \\
\hline \# - broker.stats.adv.expiration.time & \# - broker.stats.adv.expiration.time \\
\hline \# In seconds: the rest & \# In seconds: the rest \\
\hline client.ping.time $=3$ & client.ping.time $=3$ \\
\hline client.discovery.time $=10$ & client.discovery.time $=5$ \\
\hline client.discovery.publish.time $=15$ & client.discovery.publish.time $=5$ \\
\hline client.info.adv.time $=7$ & client.info.adv.time $=5$ \\
\hline client.info.adv.lifetime $=12$ & client.info.adv.lifetime $=10$ \\
\hline client.info.adv.expiration.time $=60$ & client.info.adv.expiration.time $=60$ \\
\hline client.criteriums.adv.time $=15$ & client.criteriums.adv.time $=15$ \\
\hline client.criteriums.adv.lifetime $=15$ & client.criteriums.adv.lifetime $=15$ \\
\hline client.criteriums.adv.expiration.time $=65$ & client.criteriums.adv.expiration.time $=45$ \\
\hline client.stats.adv.time $=20$ & client.stats.adv.time $=30$ \\
\hline client.stats.adv.lifetime $=5$ & client.stats.adv.lifetime $=5$ \\
\hline client.stats.adv.expiration.time $=5$ & client.stats.adv.expiration.time $=5$ \\
\hline client. stats.update.time $=5$ & client.stats.update.time $=5$ \\
\hline client.stats.max.times $=24$ & client.stats.max.times $=24$ \\
\hline discovery.room.publish.time $=50$ & discovery.room.publish.time $=5$ \\
\hline discovery.room.time $=50$ & discovery.room.time $=5$ \\
\hline pipe.adv.room.time $=50$ & pipe.adv.room.time $=5$ \\
\hline pipe.adv.broker.time $=10$ & pipe.adv.broker.time $=5$ \\
\hline pipe.adv.client.time $=10$ & pipe.adv.client.time $=5$ \\
\hline pipe.adv.lifetime $=25$ & pipe.adv.lifetime $=10$ \\
\hline pipe.adv.expiration.time $=50$ & pipe.adv.expiration.time $=50$ \\
\hline
\end{tabular}

[3] H. Bal, H. Casanova, J. Dongarra, and S. Matsuoka. Application-Level Tools. In Foster and Kesselman, eds, The Grid: Blueprint for a New Computing Infrastructure, chapter 24, 463-489. Morgan 2003.

[4] D. Brookshier, D. Govoni, N. Krishnan, and J.C Soto. JXTA: Java P2P Programming. Sams Publishing, 2002.

[5] J. Crowcroft, T. Moreton, I. Pratt, and A. Twigg. Peerto-Peer Technologies. In Foster and Kesselman, eds, The Grid: Blueprint for a New Computing Infrastructure, chapter 29, 593-622. Morgan 2003.

[6] S. Li. Early Adopter JXTA. Wrox Press Information Inc., 2003.

[7] S. Oaks, B. Traversat, and L. Gong. JXTA in a Nutshell. O'Reilly, 2003.

[8] A. Oram, ed. Peer-to-Peer: Harnessing the Power of Disruptive Technologies. O'Reilly, 1st edition, 2001.

[9] C. Shikey. What is P2P ... and what isn't. O'Reilly Network, November 2000.

[10] F. Xhafa, L. Barolli, T. Daradoumis, R. Fernandez, S. Caballé. Extension and evaluation of JXTA protocols for supporting reliable $\mathrm{P} 2 \mathrm{P}$ distributed computing. International Journal of Web Information Systems, 4(1), 121-135, 2008, Emerald Pub.

[11] F. Xhafa, L. Barolli, T. Daradoumis, R. Fernandez, S. Caballé. Jxta-Overlay: An Interface for Efficient Peer Selection in P2P JXTA-based Systems. International Journal on Computer Standards \& Interfaces, Elsevier, 2008. In Press 